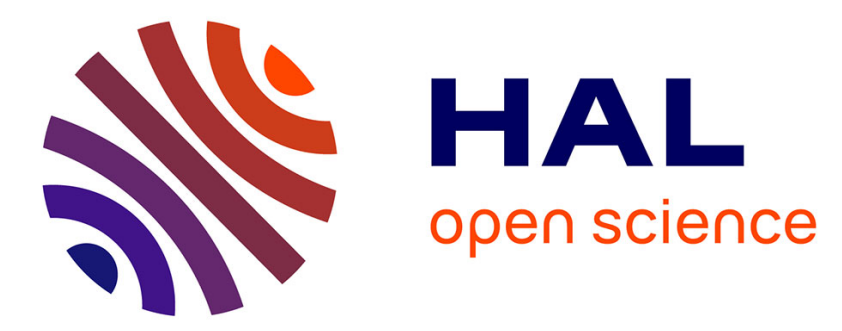

\title{
Detection and replacement of a failing node in the wireless sensors networks
}

Abdelmalek Boudries, Makhlouf Aliouat, Patrick Siarry

\section{To cite this version:}

Abdelmalek Boudries, Makhlouf Aliouat, Patrick Siarry. Detection and replacement of a failing node in the wireless sensors networks. Computers and Electrical Engineering, 2013, 10.1016/j.compeleceng.2013.10.010. 10.1016/j.compeleceng.2013.10.010 . hal-00916120

\section{HAL Id: hal-00916120 \\ https://hal.science/hal-00916120}

Submitted on 12 Dec 2013

HAL is a multi-disciplinary open access archive for the deposit and dissemination of scientific research documents, whether they are published or not. The documents may come from teaching and research institutions in France or abroad, or from public or private research centers.
L'archive ouverte pluridisciplinaire HAL, est destinée au dépôt et à la diffusion de documents scientifiques de niveau recherche, publiés ou non, émanant des établissements d'enseignement et de recherche français ou étrangers, des laboratoires publics ou privés. 


\title{
Detection and replacement of a failing node in the wireless sensors networks
}

\author{
Abdelmalek BOUDRIES ${ }^{\mathrm{a}, *}$, Makhlouf ALIOUAT ${ }^{\mathrm{a}}$, Patrick SIARRY ${ }^{\mathrm{b}}$ \\ ${ }^{a}$ University of Ferhat Abbas, Setif, Algeria. \\ ${ }^{b}$ Laboratoire Images, Signaux et Systemes Intelligents(LiSSi),University of Paris-Est \\ Creteil Val-de-Marne, Creteil 94010,France.
}

\begin{abstract}
The lifetime in a wireless network, in particular a wireless sensor network, depends strongly on the connectivity factor between nodes. Several factors can be at the origin of a connectivity rupture such as: lack of energy on a significant node level, infection of a vital node by a malevolent code, a logical or physical failure of a primary node, etc. This rupture can lead in some cases to a reconfiguration of the network by generating a prejudicial overhead or in other cases to a failure of the mission assigned to the network. In this paper, we propose a DRFN approach (Detection and Replacement of a Failing Node) for the connectivity maintenance by carrying out a replacement chain according to a distributed algorithm. Through simulation, we have shown our approach efficiency. Compared with similar work, our proposed approach consumes less energy, and improves the percentage of reduction in field coverage.
\end{abstract}

Keywords:

Wireless ad hoc network, Connectivity, Coverage, Sensors networks, Failure node.

\footnotetext{
*Corresponding author

Email addresses: abdelmalekboudries@gmail.com (Abdelmalek BOUDRIES), aliouat_m@yahoo.fr (Makhlouf ALIOUAT), siarry@univ-paris12.fr (Patrick SIARRY)
} 


\section{Introduction}

Recent technological advances have led to the emergence of the pervasive networks of small and low power devices that integrate sensors and actuators with limited on-board processing and wireless communication capabilities [1]. Nowadays, the usage of solutions based on the wireless sensor networks (WSN) [2] increases rapidly. Wireless Sensor Networks are very large collections of tiny smart sensor devices that form ad hoc distributed sensing and data management networks that collect detailed information about the ambient environment. In an usual scenario, these networks are largely deployed in areas of interest (such as inaccessible terrains or disaster places) for fine grained monitoring in various classes of applications [3, 4]. Considering the wireless sensor network generally deployed in a difficult accessing zone, the self-organization of the sensor nodes and adaptation to network dynamics, along with mutual cooperation of sensors is essential. This extends the network lifetime and reduces congestion by avoiding redundant data $[\underline{5}, 6,6,6]$. The wireless communications play a crucial role in data-processing networks. They offer open solutions to provide mobility as well as essential services where the installation of infrastructures is not possible. These networks are under active development because of their interface flexibility which offers mobility to a user. This mobility is the new considered way of communication. It generates some characteristics of the mobile environment: a frequent disconnection, a modest debit of communication, and especially the limited source of energy.

Since the majority of the low power devices have batteries with a limited lifetime and the replacement of these batteries on thousands of these devices is impossible, it is well approvable that a sensors network should be deployed with a strong density (until 20 nodes/m3 [9]) in order to extend the network lifetime. In a high density network, if all the sensors nodes function in an active mode then an excessive quantity of energy will be wasted, the data of sensors gathered are likely to be strongly correlated and redundant, and an excessive collision of packages can occur because of the simultaneous sending of packages by the sensors in the presence of some releases events. Consequently it is neither necessary nor desirable that all nodes function simultaneously in an active mode. One of the questions which emerge in such high density networks of sensors is the control of density. In [10], the authors choose the prolongation of the operating time system while keeping only a necessary set of sensors in an active mode and putting the remained 
sensors in sleep mode. Another category of approaches for the connectivity maintenance in wireless sensor networks is the restoration of the connectivity after a sensor node failure [11, 12, 13].

Supposing a wireless sensor network deployed in a difficult accessing zone. For example, in the remote harsh fields or disaster areas, sensor deployment can not be performed manually or precisely. The lifetime of this network depends strongly on the connectivity factor between its nodes. Several factors can be at the origin of a rupture of connectivity such as the lack of energy on the significant node level, infection of a vital node by a malevolent code, a logical or physical failure of a primary node etc. The failure of a sensor node can leave all its zone (or a part of it) without coverage, and can generate the partition of the network if it is a gateway node (a relay node). This means that the network will be divided into two or several small networks where some nodes can be disconnected from the entire network. This implies a loss of connectivity between the parts of the network. In addition, once deployed, sensor nodes may fail, requiring nodes to be moved to over-come the coverage hole created by the failed sensor. In this case, it is necessary to make use of mobile sensors, which can move to provide the required coverage.

Our objective is to restore connectivity after failure of a sensor node by taking into account the constraint of energy while trying to share the total consumed energy by several nodes in order to minimize the consumption of individual energy. In this paper, we propose an approach of detection and replacement of a failing node while taking into consideration the network lifetime. The aim is that the consumed total energy, for the restoration of connection, would be shared by several nodes so that the consumption of individual energy would be tiny and thus extending the global network lifetime. The evaluation results show that our approach of connectivity restoration consumes less energy and improves the percentage of reduction in field coverage.

The remainder of this paper is organized as follows: in Section 2 we will present some related work. The Section 3 is devoted to the proposed approach DRFN (Detection and Replacement of a Failing Node), followed by the Section 4 which describes the evaluation of our approach and shown its efficiency. Finally, in Section 5, we conclude the paper and we will state prospects. 


\section{Related work}

Several works are proposed for the connectivity maintenance framework in the wireless sensor networks. We classify the approaches existing in the literature in two classes (see figure 1):

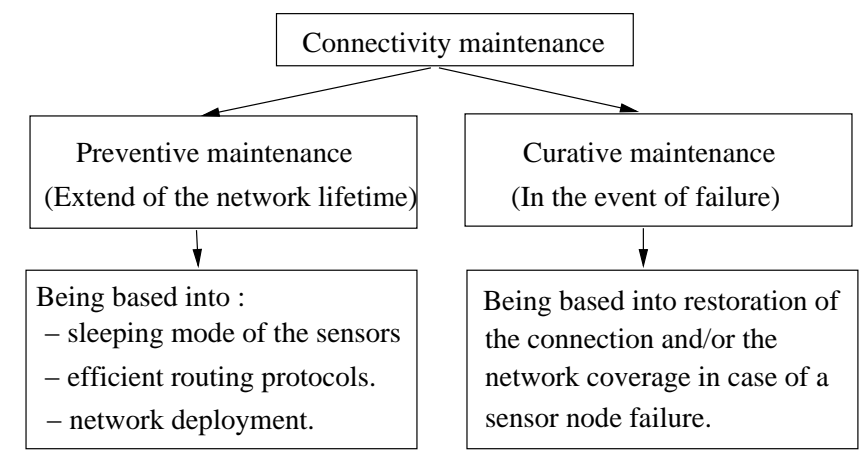

Figure 1: Classification of the solutions of connectivity maintenance

The authors of the first class try to maintain the sensors network connected longest time possible; They seek solutions to extend the lifetime of the network like [14, 15, 10, 1] which try to use, at a given time, a minimum number of sensors that ensure the connectivity and/or the network coverage, where the others are puting in sleep mode. The routing protocols also play a very significant role to prolong the network lifetime. The paths for data transfer are selected in such a way that the total energy consumed along the path is minimized [16]. Efficient routing in a sensor network requires that the routing protocol must minimize energy dissipation. To extend the network lifetime, R.V. Biradar et al. in [17] have implemented several multihop flat based routing protocols; and in [18] Liu and Wang develop a maximizing energy utilization routing protocol. To minimize energy dissipation and maximize network lifetime, Chamam and Pierre propose in [19] a novel distributed clustering algorithm where cluster heads are elected following a three-way message exchanged between each sensor and its neighbors. They demonstrate its superiority against EESH, one of the most recent cluster algorithms [20], in terms of network lifetime and ratio of elected cluster heads. The solution in this first class can also be conceived at the network deployment, to see the work of N. Aitsaadi et al. [21], the aim in this solution is to generate the best network topology in order to minimize the cost 
of deployment, to ensure the connectivity maintenance and the monitoring quality of the coverage zone and also to optimize the lifetime of the network. Liao and Wang 22] propose an asynchronous MAC protocol (AMAC) and expect to improve the problem of energy wasting and time synchronization due to sleeping schedule exchanged under the PMAC basic protocol. While the authors of the second class work on the connectivity maintenance in case of failure of a sensor node [11, 12, 13], and try to solve the problem of connectivity in the case of the network partitioning and/or the covering of the monitoring zone problem. We classify our work in the second class (Curative maintenance).

In DARA approach [11] the detection of a failing node can not require an adjustment in the network topology if the node does not divide the network into parts. It means that the coverage zone of the failing node can remain without coverage if the failure of this node in question doesn't partition the network and that even if there are redundant nodes in the neighbourhood of the failing node. DARA is focused on the connectivity maintenance without being concerned with the network coverage. In this approach, a substitute of the failure or the moved node leaves its zone definitively without coverage even if no other node can replace it afterwards. In terms of coverage, this node can be considered failing by leaving its zone without coverage.

In the paper of N. Tamboli et al. [12], the replacement of the failed node is done only by its direct neighbors. The exhaustion of energy due to the repeated physical movements of these nodes is an obvious concern to be taken into account. If we limit the replacements only to the direct neighbors of this node, we probably cause the failure of several nodes in a reduced time and put in danger the entire network. However, if we want to extend the lifetime of the sensors network, the replacement function of the failed node must be shared by several neighbors using replacements chain. This replacements will continue until arriving at a node which its coverage range is completely covered by its neighbors (in this case, the algorithm will finish) or arrive at an extremity node less significant in terms of connectivity of the entire network (in this case, if we don't take into account the network coverage, the algorithm will finish. But if we take into account the network coverage, the algorithm will continue to be executed).

The suggested method in [13], called AOM, consists of three steps such as: the network setup, monitoring the neighbors status and detecting failure of actors, and finally restoring the network after actor failure. After the network is deployed, the following steps are taken: - determining the 
critical node and the connectivity dominating set CDS), and - determining restoration policy for each critical actor. For determining the critical node and the connectivity dominating set, the authors have respectively used the method of localization suggested by Jorgic et al. 23. which is not exact but it detects quickly the critical nodes, and the CDS method suggested by Dai and $\mathrm{Wu}[24$. The authors have used a proactive policy in order to restore the network in the event of critical actor failure. When critical nodes in the network are determined, each critical node requests its neighbors to send the maximum distance that they can move towards the critical node without being disconnected from their other neighbors. If a critical node fails, the network is partitioned into two or more sub networks. By applying the method proposed, they can determine to which partition each neighbor of the cut vertex node belongs. If there is a dominate node $v$ in the neighbor of the cut vertex, a message is sent to $v$ to notify that it must replace the cut vertex in the case of failure. If there is any such actor, the maximum allowable movement of each neighbor of the cut vertex is calculated. It must be noted that some neighbors of the cut vertex may belong to one partition. Therefore, from each partition, the nearest neighbors to cut vertex are selected and a request message is sent to each of them to send their maximum movement. Each neighbor of the cut vertex that receives the message calculates the location of the furthest actor in its own neighbor. Therefore, it would determine how much each neighbor of the cut vertex could move towards the cut vertex without violating the connectivity of the network. At the critical node, after receiving all the messages from neighbors, it checks whether there is a neighbor $v$, whose maxMovement covers the location of the cut vertex. This condition occurs if $v$ does not have any other neighbor except the cut vertex, so it is a good candidate for replacement. If this criterion is held, $v$ is responsible for handling the failure of the actor and it would replace the cut vertex in case of failure. If this criterion is not held, the cut vertex calculates whether the network could be reconnected if neighboring actors move by their maxMovement. Wang et al. in [25] define the problem of relocation of sensors in the case of failure, and propose a solution of relocation in two phases. The first phase consists in identifying the redundant sensors by proposing the solution "quorum of grids" to locate quickly these redundant sensors. The second phase is the replacement in the target place by using the cascaded movement. What we reproach to this approach is that if the network application requires separated measurements by a very short time lapse for each sensor node then this solution of two phases can prove to 
be expensive in terms of time for the mission of the entire networks.

Our proposed approach, called DRFN (detection and replacement of a failing node), take into consideration the network lifetime, we want that the consumed total energy for the restoration of connection would be shared by several nodes so that the consumption of individual energy would be tiny and thus extending the global network lifetime.

\section{DRFN : Detection and replacement approach of a failing node for connectivity maintenance in the wireless sensors networks}

If a sensor node $S n$ fails (because of a lack of energy on the level of its battery for example), then one of its neighbors $n_{i}$ moves to replace it and ensures the functions of this failing node $S n$ (such as the coverage of its zone and the the connectivity maintenance with its neighbors). One of the neighbors of the node $n_{i}$ goes, in its turn, to take the place left by the node $n_{i}$ and will ensure its functions. The same process of replacement will continue until arriving:

- at a node where its zone is completely covered by its neighbors (see the first class in figure 1 where the redundant nodes are put in sleep mode); or

- to arrive at a node which does not have any other neighbor other than the node subject for the replacement. In this case, this node must ensure its functions and the functions of the replaced node in intermittency by making back and forth between its place and the place of the replaced node until its weight decreases compared to the other neighbors of the replaced node.

The idea is to imply in the replacement a node which has a potential energy higher than a node which has a low potential energy. The number of neighbors and the distance between the sensors can also be a significant criteria. The implication of several nodes permits to share the energy consumption and thus to extend the global network lifetime.

In the case of presence of several neighbors of the failure node or the node elected substitute, what is the process to follow to elect a substitute? Several solutions can be considered: 
- Supposing the one which has less neighbors (less charged) to be elected. In this case, if the node is weak in terms of energy, it will be preferable to take another node with a higher potential energy;

- Supposing that we opt now to the election the one which has a higher potential energy. In this case, if the node has a great number of neighbors, it means that it is a very significant relay node in terms of connectivity. It will be then preferable to support the election of another node with a lower number of neighbors.

For that, a compromise between the potential energy and the number of neighbors is essential, without forgetting the distance separating it from the failed node; Because if this distance is large, exhausted energy due to the physical movement of this node is high and the probability that this node would be elected must decrease, which decreases its weight. For that, we define the weight of a node $(w n)$, compared to his percentage of energy and his number of neighbors, as well as the distance separating it from the failed node, in the following way:

$$
w n=\frac{\% \text { energy }}{\alpha \times \text { numberofneighbors }+\beta \times \text { distance }}
$$

Where :

- distance is the distance separating it from the failed node,

- $\alpha, \beta$ are empirical variables (fixed to 1 ).

Thus, if the percentage of energy decreases, the weight of the node decreases and its probability that it would be elected decreases. In the same way, if the number of neighbors increases (which explains the overload of this node), the weight of the node decreases (see figure 2) and its probability that it would be elected decreases too. Same thing, each time the distance is large, the consumed energy due to the displacement of the node is large and the weight of this last one decreases, thus its probability to be elected decreases. 


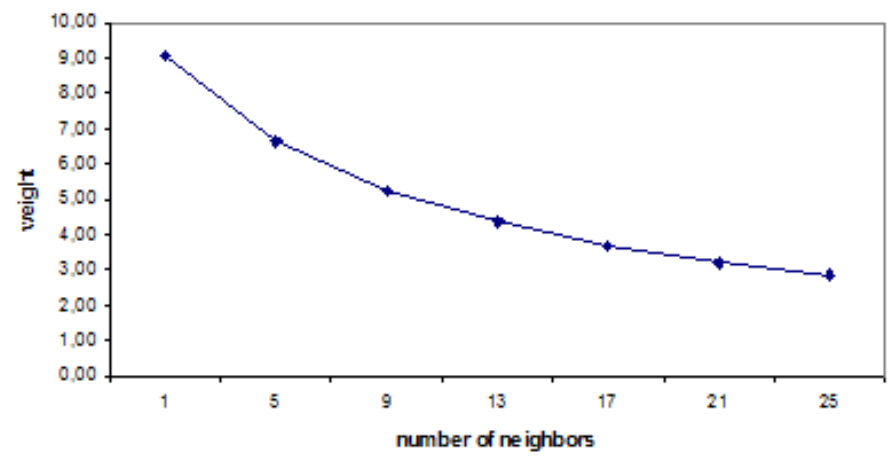

Figure 2: variation of the weight of the sensors node according to the number of direct neighbors (\% energy and distance are fixed)

The detection process of a failing node is illustrated in algorithm 1 (Detect-fail()) where we describe the instructions which a sensor node must execute to detect a failing neighbor. After a well defined waiting, the sensor node sends a small message detect then waits a certain duration before judging that the sensor node is failing. After this duration, which is equal to the time of go and back of the message plus the processing time, if the sensor node transmitting the message detect does not receive an answer then it considers the recipient node of the message failing. And the process is always repeated after a well defined waiting.

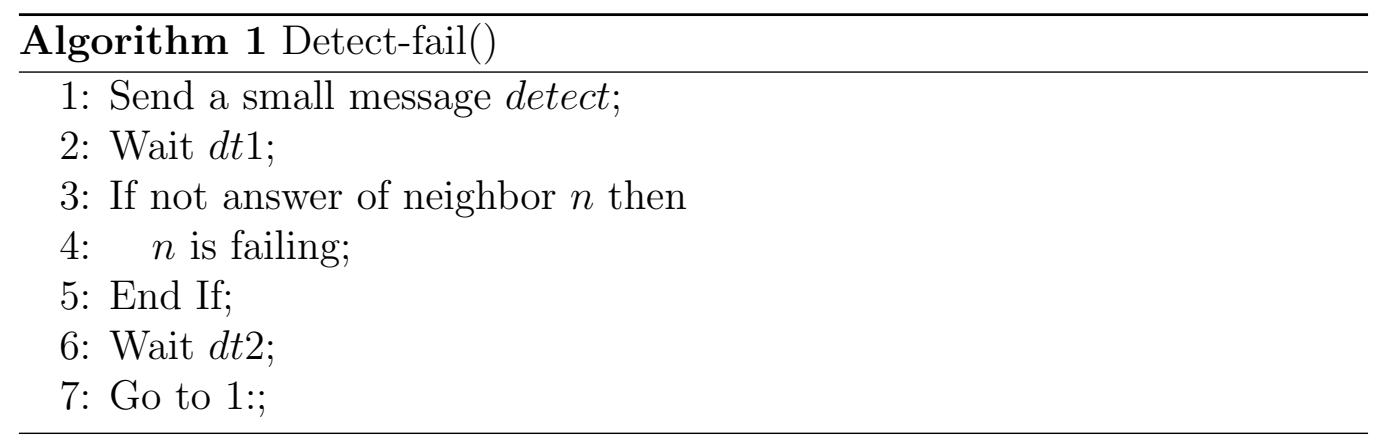

Where: $d t 1$ represents the latency of the answer before judging that the sensor is failing, and $d t 2$ represents the separating time of two detect messages. 
Proposition 1. If the size of the message increases then the latency $d t 1$ to judge that the sensor is failing increases. And when the rate of transmission increases, the latency $d t 1$ decreases.

Proof. $d t 1$ is a sufficient time so that a sensor $s$ receives an answer: it is equal to the time sum of both go and back of a message (twice the time of transmission $t_{\text {trans }}$ ) of the sensor $s$ to the farthest direct neighbor and to the processing time $\left(t_{\text {trait }}\right)$ of a message by the sensor which receives it (see formula 2).

$$
d t 1=2 \times t_{\text {trans }}+t_{\text {trait }}
$$

We have also :

$$
t_{m s g}=d_{\text {trans }} \times t_{\text {trans }}
$$

Where $d_{\text {trans }}:$ is the debit of transmission

$t_{m s g}:$ is the size of the message sent.

The formula 3 implies that :

$$
t_{\text {trans }}=\frac{t_{m s g}}{d_{\text {trans }}}
$$

We replace formula 4 in formula 2 , we will have:

$$
d t 1=\frac{2 \times t_{\text {msg }}}{d_{\text {trans }}}+t_{\text {trait }}
$$

Thus in formula 5 we notice that if the size of the message $t_{m s g}$ increases then the latency $d t 1$, to judge that the sensor is failing, increases; And when the transmission debit $d_{\text {trans }}$ increases then the latency $d t 1$ decreases.

The process of replacement is illustrated, in a general way, in algorithm 2 (repl-fail()) where we have described the reaction of a given sensor node towards a detection of a failing sensor node or a moving sensor node, and the reaction of a sensor node if it is elected substitute. The algorithm is executed if there is a detection of a failing or moving node and when a sensor node is elected substitute. If a node is elected substitute, it moves initially to provide the functions of the failing or moving node and it checks if its list of direct neighbors is empty or not. If it is not empty, it does not do anything because its direct neighbors will elect a substitute to replace it. However, if its list of direct neighbor is empty, it will be obliged to return to its place after a well defined quantum of time. 


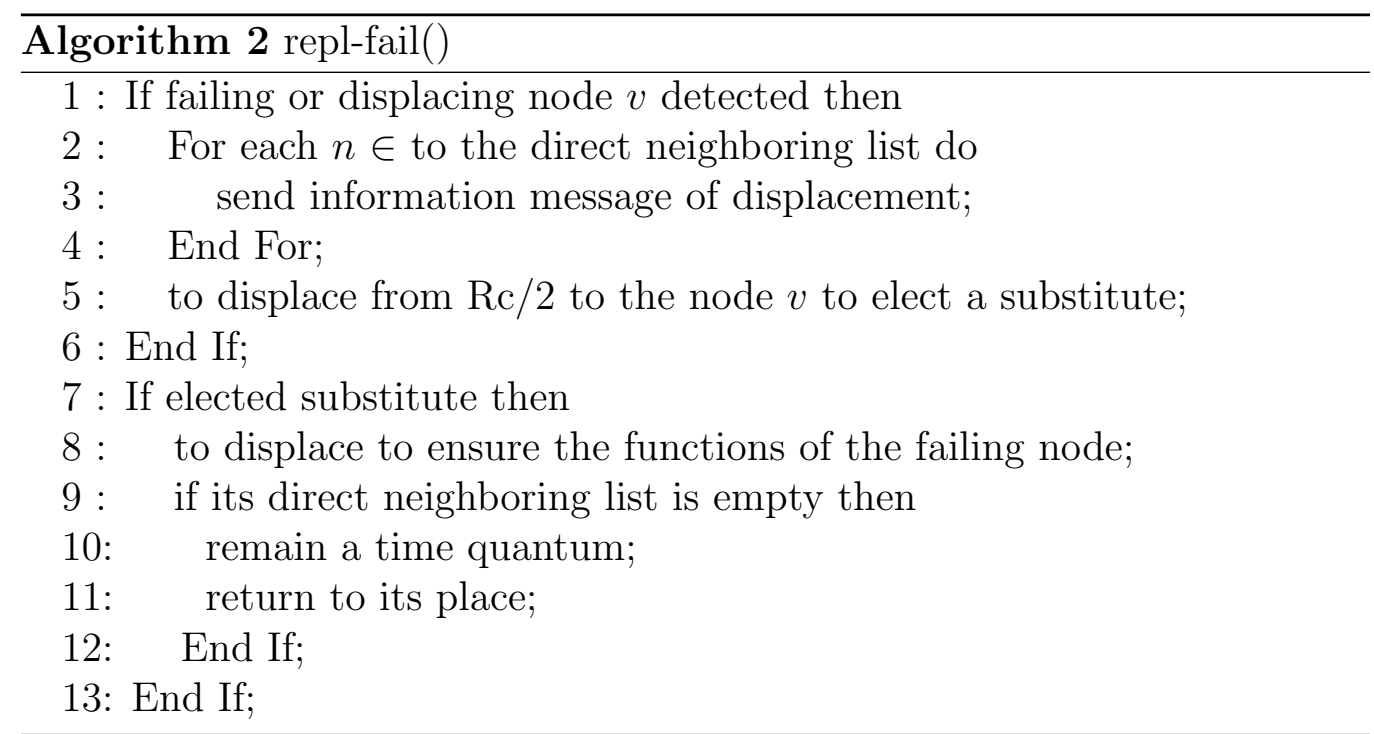

Proposition 2. Considering a wireless sensor network where all the sensors have the same communication ray Rc. The displacement of the close nodes, towards the failing node, from a distance of Rc/2 to elect a substitute is a sufficient condition to connect and exchange messages.

Proof. Considering two sensors nodes $s n_{1}$ and $s n_{2}$ the farthest neighbors of the failing node $f$ located at the center of the trigonometrically circle of figure 3 , we have then:

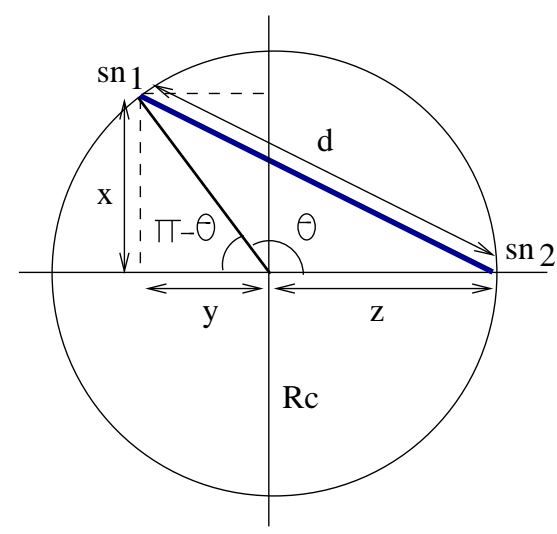

Figure 3: Outdistance between two neighbors of a given node 


$$
d^{2}=x^{2}+(z+y)^{2}
$$

We have too :

$$
\begin{gathered}
x=\sin (\Pi-\theta) \\
z=1 \\
y=\cos (\Pi-\theta)
\end{gathered}
$$

By replacement we have:

$$
d^{2}=\sin (\Pi-\theta)^{2}+(1+\cos (\Pi-\theta))^{2}
$$

Which implies that:

$$
d=\sqrt{\sin (\Pi-\theta)^{2}+(1+\cos (\Pi-\theta))^{2}}
$$

In formula 11, $d$ reaches its maximum when $\cos (\Pi-\theta)$ is equal to 1 .

This implies that $\Pi-\theta=0 \Rightarrow \Pi=\theta$. Then these nodes are on the same line with the failing node $f$ (see figure 4 ).

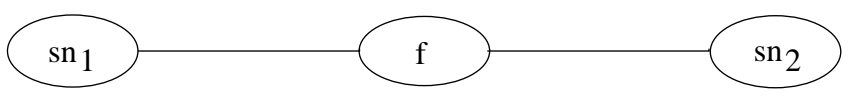

Figure 4: max Outdistance between two neighbors of a given node

So the longest distance we can have between the neighbors of node $f$ is equal to $2 \times$ Rc (i.e. the distance separating $s n_{1}$ from $s n_{2}$ is of $2 \times \mathrm{Rc}$ ). Let us suppose now that both the nodes $s n_{1}$ and $s n_{2}$ move from Rc/2 towards $f$ so they travel Rc distance. This implies that the new distance between $s n_{1}$ and $s n_{2}$ is equal to $2 \times \mathrm{Rc}-\mathrm{Rc}=\mathrm{Rc}$, it is exactly the communication range of these sensors nodes. Thus they will be connected and they can communicate between them. 


\section{Scenario example (Figure 5)}

\section{Case 1: single failure}

If the sensor node $s n_{6}$ fails then the set of nodes $\left\{s n_{1}, s n_{2}, s n_{5}, s n_{9}\right\}$ will be disconnected from the network.

In the same way, if the node $s n_{7}$ fails then is the set $\left\{s n_{3}, s n_{4}, s n_{8}\right\}$ which will be disconnected from the remaining of the network.

And if it is the nodes $s n_{10}$ or $s n_{11}$ which fails then it is the set $\left\{s n_{13}, s n_{14}\right\}$ or the set $\left\{s n_{12}, s n_{15}, s n_{16}\right\}$, which will be respectively, disconnected from the remaining of the network. Supposing, now, that all the nodes have an equal potential energy and the distance between the nodes is the same, and that the node $s n_{10}$ fails;

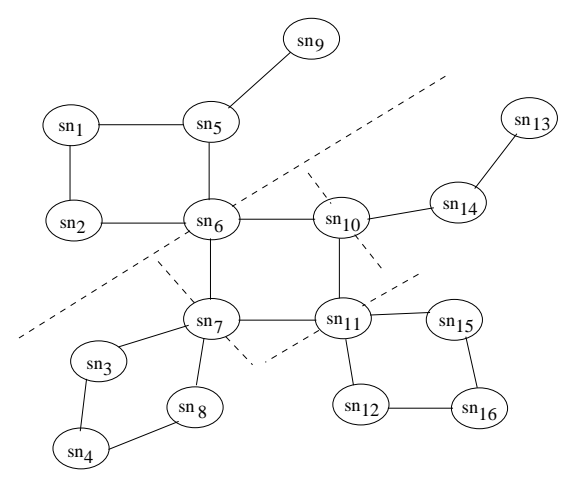

Figure 5: Connected network of mobile sensors nodes

- Among its neighbors, the node $s n_{14}$ has a less number of neighbors, thus it will be elected to replace $s n_{10}$,

- $s n_{13}$ is the only neighbor of $s n_{14}$. Then it will be elected to replace it,

- The node $s n_{13}$ does not have any other neighbor than the node $s n_{14}$, thus it must ensure its functions and those of the node $s n_{14}$ by making back and forth between its zone and the zone left of the node $s n_{14}$ at a well defined time quantum. Each time $s n_{13}$ returns in its zone, it negotiates the election with $s n_{14}$. Once the weight of the node $s n_{13}$ decreases compared to the weight of the node $s n_{14}$, this node $s n_{14}$ in its turn will participate in the replacements. 


\section{Case 2: multiple failures 1}

If the sensor nodes $s n_{6}$ and $s n_{7}$ fails, the sets of nodes $\left\{s n_{1}, s n_{2}, s n_{5}, s n_{9}\right\}$ and $\left\{s n_{3}, s n_{4}, s n_{8}\right\}$ will be disconnected from the network.

- Among its neighbors, the node $s n_{2}$ has a less number of neighbors, thus it will be elected to replace $s n_{6}$,

- $s n_{1}$ is the only neighbor of $s n_{2}$, it will be elected to replace it,

- The nodes $s n_{3}$ and $s n_{8}$ have the same number of neighbors. In this case, the node which has the smaller index (node $s n_{3}$ ) will be elected to replace the failed node $s n_{7}$

- $s n_{4}$ is the only neighbor of $s n_{3}$, it will be elected to replace it,

The same process continues until arriving at a node which its coverage range is completely covered by its neighbors or arrive at an extremity node less significant in terms of connectivity of the global network.

\section{Case 3: multiple failures 2}

Supposing now the failure of the nodes $s n_{6}$ and $s n_{8}$. The problem in this case is: the node $s n_{7}$ is the neighbor of 2 failed nodes, it must participate to elect a substitute for only one of this failed nodes. If this multiple failures are detected at different time, the node $s n_{7}$ participates to elect the first detected failure. But if these multiple failures are detected at the same time, it must participate to elect a substitute for the failed node which are the smaller index.

The same process continues as described in case 1 and case 2 .

The failing of some nodes (for example extremity nodes) does not effect the network connectivity, but have an effect on the network coverage. For that, our replacement algorithm must be executed for any failed node. Thus we will be able to guarantee the network connectivity and coverage at the same time.

\section{Evaluation}

We evaluate our approach compared to the $C^{3} R$ approach [12, taking as metric the distance moved, the number of nodes implied for the restoration of connectivity, the percentage of reduction in coverage, and the generated messaging overhead. 


\subsection{Simulation settings}

The simulations results are given by our simulator developed with java language. In the experiments, a set of mobile sensor nodes are initially placed at random in a $1000 \times 1000 \mathrm{~m}^{2}$ area to form a WSN. All nodes are assumed to have the same communication and sensing ranges. We measure: First, the number and the difference of displacements in function of time for both $D R F N$ and $C^{3} R$ approaches. Second, the average percentage reduction in field coverage for node population of 75 and 200 by varying the sensing range. And finally, the total packets exchanged in function of communication range.

4.2. The moved distance and the number of nodes implied for the restoration of connectivity

Considering the following assumptions:

- All nodes are mobile;

- The displacement time of a node to another neighbor node is 10 seconds;

- The network application requires measurements each 30 seconds for each sensor;

- The distances between the neighbors nodes are equal to $d$;

- The sending time, reception and treatment of messages as well as the election time of substitute are neglected.

Let us suppose the failure of the node $s n_{6}$ in figure 5 : 
Table 1: Comparison between $C^{3} R$ and our approach $D R F N$ (Detection and Replacement of a Failing Node)

\begin{tabular}{|c|c|c|c|c|c|}
\hline \multicolumn{3}{|c|}{$C^{3} R$} & \multicolumn{3}{|c|}{$D R F N$} \\
\hline Displacement & $\begin{array}{l}\text { Displacement } \\
\text { time }\end{array}$ & $\begin{array}{l}\text { Stay } \\
\text { time }\end{array}$ & Displacement & $\begin{array}{l}\text { Displacement } \\
\text { time }\end{array}$ & $\begin{array}{l}\text { Stay } \\
\text { time }\end{array}$ \\
\hline $\begin{array}{l}s n_{10} \rightarrow s n_{6} \\
s n_{10} \rightarrow s n_{10}\end{array}$ & $\begin{array}{l}10 \\
10\end{array}$ & 10 & $s n_{10} \rightarrow s n_{6}$ & 10 & $\begin{array}{l}10 \\
10\end{array}$ \\
\hline \multicolumn{3}{|c|}{10 seconds wait } & \multirow[t]{2}{*}{$s n_{11} \rightarrow s n_{10}$} & \multirow[t]{2}{*}{10} & \multirow[b]{2}{*}{$\begin{array}{l}10 \\
10\end{array}$} \\
\hline$s n_{5} \rightarrow s n_{6}$ & 10 & \multirow[t]{2}{*}{10} & & & \\
\hline$s n_{5} \rightarrow s n_{5}$ & 10 & & \multirow[t]{3}{*}{$s n_{12} \rightarrow s n_{11}$} & \multirow[t]{3}{*}{10} & \multirow{3}{*}{$\begin{array}{l}10 \\
10\end{array}$} \\
\hline \multicolumn{3}{|c|}{10 seconds wait } & & & \\
\hline \multirow{2}{*}{$\begin{array}{l}s n_{2} \rightarrow s n_{6} \\
s n_{2} \rightarrow s n_{2}\end{array}$} & \multirow[t]{2}{*}{10} & \multirow[b]{2}{*}{10} & & & \\
\hline & & & \multirow[t]{2}{*}{$s n_{16} \rightarrow s n_{12}$} & \multirow[t]{2}{*}{10} & \multirow{2}{*}{$\begin{array}{l}10 \\
10\end{array}$} \\
\hline \multicolumn{3}{|c|}{10 seconds wait } & & & \\
\hline $\begin{array}{l}s n_{7} \rightarrow s n_{6} \\
s n_{7} \rightarrow s n_{7}\end{array}$ & $\begin{array}{l}10 \\
10\end{array}$ & 10 & $s n_{15} \rightarrow s n_{16}$ & 10 & $\begin{array}{l}10 \\
10\end{array}$ \\
\hline
\end{tabular}

Where $s n_{i} \rightarrow s n_{j}$ means the displacement of the node $s n_{i}$ from its place towards the place of the node $s n_{j}$, and $s n_{i} \rightarrow s n_{i}$ means return of $s n_{i}$ towards its initial place.

We take, for the analysis, a period of time of 150 seconds. This is represented in table 1. During this period, the number of displacements, represented in figure 6, carried out with the $C^{3} R$ approach is 8 , which results that the total travelled distance is of $8 d$ ( $d$ is the distance between the neighbors nodes), compared to 5 displacements $(5 d)$ with $D R F N$ approach. The number of nodes implied in the replacement is 5 for $D R F N$ approach, to 4 nodes for the $C^{3} R$ approach (it is exactly the number of direct neighbors of the failing node). 


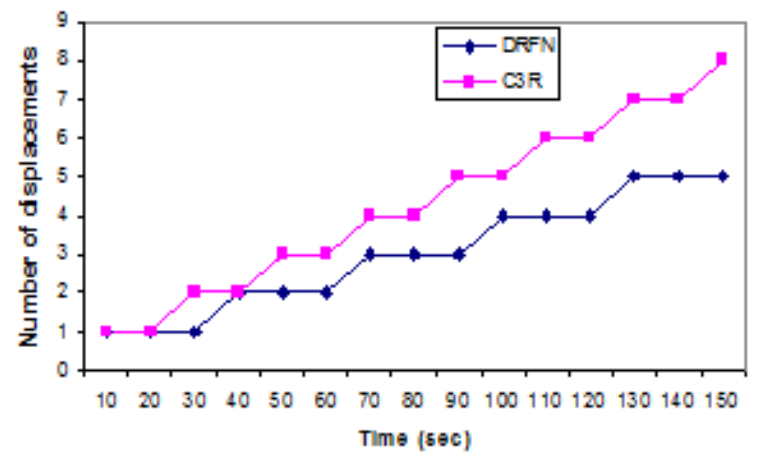

Figure 6: Displacements number of sensors per time unit

The figure 7 shows the difference of the displacements number between $D R F N$ and $C^{3} R$ per time unit. We notice that the difference of the displacement number between $D R F N$ and $C^{3} R$ extends with time.

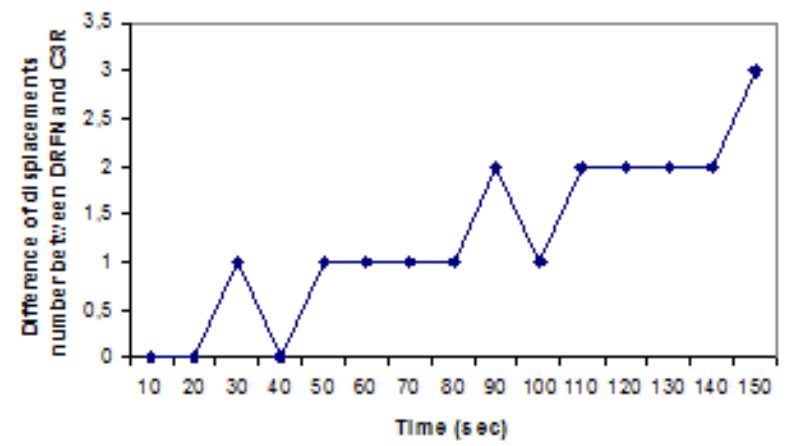

Figure 7: Difference of displacements number between $D R F N$ and $C^{3} R$ per time unit

The figure 8 shows the result of simulation according to the example of table 1 and the stated assumptions. It shows that, for each period of 120 time units, the formula 12 is checked :

$$
n b r_{-} d i s p l\left(C^{3} R\right)=\frac{3}{2} \times n b r_{-} \operatorname{displ}(D R F N)
$$




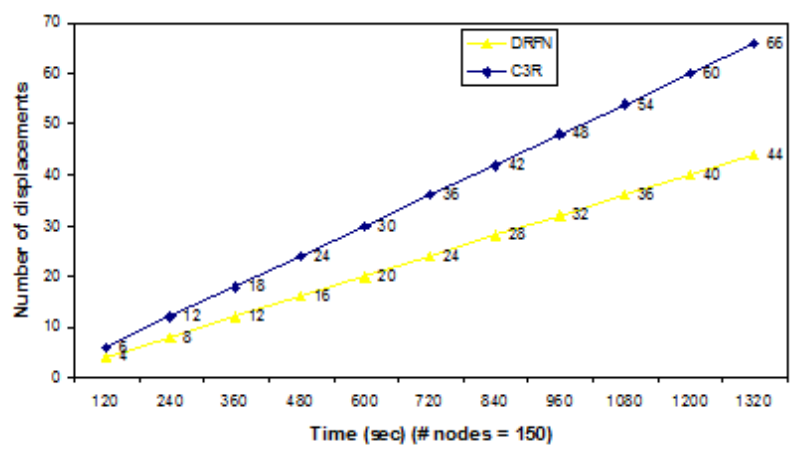

Figure 8: Displacements number per time unit for a period time of 1320 units

It results that the consumed total energy, due to displacement with the $C^{3} R$ approach, is equal to $3 / 2$ times of that consumed to displacement with $D R F N$ approach during the same period of time. This implies that the energy consumed individually by each node implied in the replacement with $D R F N$ approach is much lower than that consumed individually by each node implied in the replacement with the $C^{3} R$ approach. Because the number of nodes implied in the replacement with $D R F N$ approach is much higher than that in the $C^{3} R$ approach.

The results illustrated in figures 6, 7 and 8 reinforce the importance of our proposed DRFN approach compared to $C^{3} R$ approach in terms of displacement for the replacement of a failing node. Given this, our $D R F N$ approach consumes less energy than the $C^{3} R$ approach to replace a failing node. 


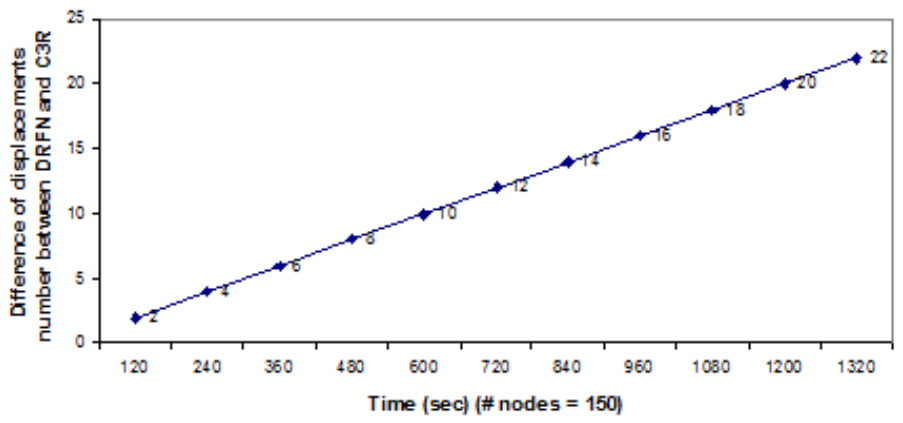

Figure 9: The difference of displacements number between $D R F N$ and $C^{3} R$ for a period time of 1320 units

The difference of displacements number between $D R F N$ and $C^{3} R$ approaches, represented in figure 9 , is an arithmetic suite of reason 2 for the multiple time values of 120 . This difference reinforces the importance of our $D R F N$ approach for the replacement of a failing node.

\subsection{Percentage of reduction in coverage}

This metric is significant because it gives us the percentage of reduction in field coverage after the execution of the connectivity restoration process when a sensor node fail.

Reduction in coverage is measured by averaging the entire field coverage recorded at different time instances over the lifetime of the network. The entire field is divided into grid points and coverage is calculated using the approximation method of calculating coverage as described in [12]. 


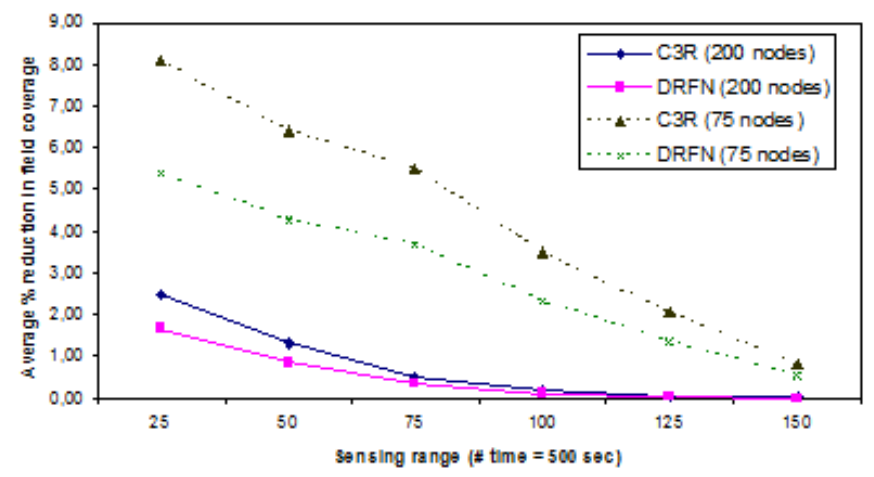

Figure 10: Reduction in field coverage for node population of 75 and 200 by varying the sensing range

Figure 10 shows the impact of the connectivity restoration on the network coverage, measured in terms of percentage of reduction in field coverage relative to the pre-failure level. $D R F N$ could significantly minimize the loss in coverage and this by minimizing the displacements number during the execution of the connectivity restoration process. During the time of displacement, the displacement sensor node leaves its zone without coverage. Thus if the displacements number increases, the percentage of reduction in field coverage increases. This explains the result in figure 10 where we have always less higher percentage of reduction in field coverage in our DRFN approach than in the related $C^{3} R$ approach. In fact to restore the coverage after a sensor node failure, our $D R F N$ approach uses less displacements than the $C^{3} R$ approach. When the sensors nodes are deployed with a great density (case of 200 nodes), the percentage of reduction in field coverage is less higher than when the sensors nodes are deployed with small density (case of 75 nodes). When the sensors nodes are deployed with a great density, the distance between the neighbors nodes is smaller, then the displacement time to replace a failing sensor node is smaller. This is explained, in figure 10, by the diminution of the reduction percentage in field coverage in the case when the sensors nodes are deployed with a small density.

\subsection{Additional overhead}

Like many works, our approach generates an additional overhead due to the detection of the failing node and the substitute election by the direct 
neighbours of the failing or displacing node.

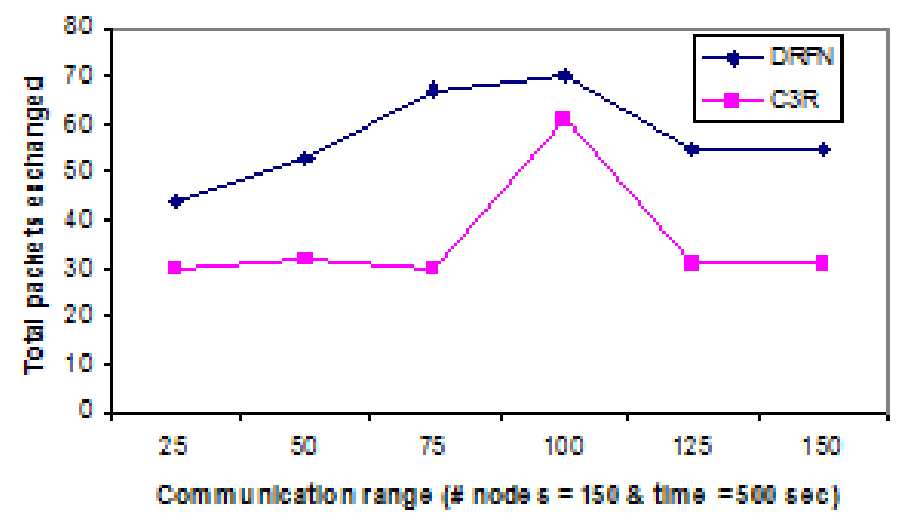

Figure 11: Messaging overhead

We notice in figure 11 that our $D R F N$ approach generates little more messages than the $C^{3} R$ approach. This is explained by the approach policy where the stages to follow to replace a failing node differ. In the $C^{3} R$ approach, the replacement of the failed node is done only by its direct neighbors and the messages for the election of a substitute node are only interchanged between the direct neighbors. But in $D R F N$ approach, the node elected for the replacement must also be replaced and the direct neighbors of the replacing node must also move and interchange messages to elect a substitute for the moved node. So, the messaging overhead is a little bigger in DRFN approach than in $C^{3} R$ approach.

The number of displacements with our DRFN approach is inferior to the number of displacements with $C^{3} R$ approach. For 120 seconds, we note 4 displacements with DRFN approach compared to 6 displacements with $C^{3} R$ approach. The number of displacements increases with time. For 1320 seconds, we note 44 displacements with $D R F N$ approach to 66 with $C^{3} R$ approach. This explains that the quantity of consumed energy with $D R F N$ approach is inferior to the quantity of consumed energy with $C^{3} R$ approach. Compared to the reduction of the field coverage, our DRFN approach reduces less the field coverage. For 75 nodes and a sensing range of 25 meters, our DRFN approach reduces of $5.40 \%$ the field coverage during 500 seconds 
compared to $8.10 \%$ with the $C^{3} R$ approach. The percentage of reduction of the field coverage decreases for the two approaches by increasing the sensing range. For 150 meters, our approach reduces of $0.53 \%$ the field coverage during 500 seconds to $0.80 \%$ with $C^{3} R$ approach. By increasing the nodes density the percentage of reduction of the field coverage decreases. For 200 nodes and a sensing range of 25 meters, our DRFN approach reduces of $1.67 \%$ the field coverage to $2.50 \%$ with $C^{3} R$ approach. For a sensing range of 150 meters, our approach reduces of $0.01 \%$ the field coverage to $0.02 \%$ with $C^{3} R$ approach. Compared to these values, we conclude that the percentage of the field coverage reduction decreases with the increasing of the sensing range or with the increasing of the nodes density, and our approach reduces it less. Compared to the number of exchanged packets, we note that our DRFN approach generates little more packets than the $C^{3} R$ approach, because the number of nodes implied for the replacement of the failing node with our DRFN approach is superior to the number of nodes implied for the replacement with the $C^{3} R$ approach.

\section{Conclusion}

The wireless sensors networks are generally deployed in hard and difficult access environments where the breakdowns or failures of sensors nodes are possible. These nodes failures can harm the connectivity of the entire network. In other words, the network can be partitioned where some nodes can be disconnected from the global network. This implies a loss of connectivity between the parts of this network.

To answer this connectivity loss, we have proposed a detection and replacement approach of a failing node, by carrying out replacements chain following a distributed algorithm. Our solution is based on two algorithms: the first one consists in detecting the failing node based on predefined lapses of time. After each lapse, node that does not answer is considered failed. The second algorithm is to replace the detected failing node by carrying out replacements chain following a distributed algorithm. The principal goal of this approach is to restore the network connectivity by exploiting the sensors mobility taking into account the energy constraint. The idea is to share the consumption of energy, necessary to the connectivity restoration, with several sensors to minimize the early failures of the sensor nodes, and thus to prolong the lifetime of the entire network. For that, we have evaluated our $D R F N$ approach with a $C^{3} R$ related approach. The simulation results show 
well that during the same period, the rate of consumed energy with $D R F N$ approach is lower than the rate of energy consumed with the $C^{3} R$ approach. And also the rate of consumed energy with $D R F N$ approach is shared by more nodes than with the $C^{3} R$ approach. This explains that the quantity of the consumed individual energy by the sensors nodes in our $D R F N$ approach is much smaller than in the $C^{3} R$ approach. Compared to the network coverage, in our DRFN approach, the percentage of reduction in field coverage is lower than in related $C^{3} R$ approach.For 75 nodes and a sensing range of 25 meters during 500 seconds, our DRFN approach reduces less of $2.7 \%$ the field coverage compared to $C^{3} R$ approach. This percentage decreases by increasing both the sensing range (for 150 meters, we note $0.27 \%$ ) and the nodes density (for 200 nodes, considering a sensing range of 25 meters, we note $0.83 \%$. However, for a sensing range of 150 meters, we note $0.01 \%$ ). To restore the coverage after sensor node failure, our $D R F N$ approach uses less displacements than $C^{3} R$ approach. We note that the difference of the displacements number is 22 displacements for 1320 seconds. The necessary energy quantity for these 22 displacements is stored in our DRFN approach, and efficiently contributes to prolong the lifetime of the network.

The evaluation results show that our approach of connectivity restoration consumes less energy, and improves the percentage of reduction in field coverage. The optimized values of both $\alpha$ and $\beta$ in formula 1 is an important problem that should be discussed in the future work.

\section{References}

[1] H. Zhang and J. C. Hou, Maintaining sensing coverage and connectivity in large sensor networks, Ad Hoc and Sensor Wireless Networks journal (AHSWN), 1(1-2), (2005), pp. 89-123.

[2] J. Yick, B. Mukherjee, and D. Ghosal, Wireless sensor network survey, In Computer Networks, 52(12), (2008), pp. 2292-2330.

[3] I.F. Akyildiz, W. Su, Y. Sankarasubramaniam, E. Cayirci, Wireless sensor networks: a survey, In journal of Computer Networks 38 (2002) 393-422.

[4] A. Boukerche, R. Pazzi, and R. Araujo, A supporting protocol to periodic, event-driven and query-based application scenarios for critical conditions surveillance, In First International Workshop on Algorithmic Aspects of 
Wireless Sensor Networks (ALGOSENSORS), Springer, Lecture Notes on Computer Science, vol. 3121, 2004, pp. 137-146.

[5] Bein Doina, Self-configuring, self-organizing, and self-healing schemes in wireless sensor networks, In Misra Sudip, Woungang Isaac, Misra Subhas C, editors. Guide to Wireless Ad Hoc Networks, Computer Communications and Networks, London: Springer-Verlag, 2009, pp. 293-304.

[6] Chen Benjie, Jamieson Kyle, Balakrishnan Hari, and Morris Robert, Span: an energy-efficient coordination algorithm for topology maintenance in ad hoc wireless networks, ACM Wireless Networks Journal, 8(5), (2002), pp. 481-494.

[7] R. Krishnan, and D. Starobinski, Message-efficient self-organization of wireless sensor networks, IEEE WCNC, New Orleans, 2003, pp. 16031608.

[8] S. Misra and A. Jain, Policy controlled self-configuration in unattended wireless sensor networks, Journal of Network and Computer Applications, 34(2011), pp. 1530-1544.

[9] E. Shih, S. Cho, N. Ickes, R. Min, A. Sinha, A Wang and A Chandrakasan, Physical layer driven protocol and algorithm design for energyefficient wireless sensor networks, In Proceedings of MobiCom'01, 7th annual international conference on Mobile computing and networking, Rome, Italy, July 2001.

[10] F. Ye, G. Zhong, S. Lu and L. Zhang, Peas: A robust energy conserving protocol for long-lived sensor networks, In The 23nd International Conference on Distributed Computing Systems (ICDCS), Providence Rhode Island, 2003.

[11] Ameer Ahmed Abbasi, Mohamed Younis, and Kemal Akkaya, Movement-Assisted Connectivity Restoration in Wireless Sensor and Actor Networks, In IEEE Transactions on parallel and distributed systems, 20(9), (2009), pp. 1366-1379.

[12] N. Tamboli, and M. Younis, Coverage-aware connectivity restoration in mobile sensor networks, Journal of Network and Computer Applications, 33(4), (2010), pp. 363-374. 
[13] A. Zamanifar, O. Kashefi and M. Sharifi, AOM : An efficient approach to restore actor-actor connectivity in wireless sensor and actor networks, In International Journal of Computer Networks \& Communications, 1(1), (2009), pp. 61-72.

[14] A. Makhoul, R. Saadi, and C. Pham, Surveillance vidéo sur réseaux de capteurs sans-fils: ordonnancement adaptatif avec prise en compte de la criticité, In CFIP (Colloque francophone sur l'ingénierie des protocoles), Strasbourg, France, 2009.

[15] D. Tian and N. D. Georganas, A coverage-preserving node scheduling scheme for large wireless sensor networks, In First ACM International workshop on Wireless Sensor Networks and Applications (WSNA), Georgia, USA, 2002, pp. 32-41.

[16] V. Kumar, S. Jain, and S. Tiwari, Energy Efficient Clustering Algorithms in Wireless Sensor Networks: A Survey, IJCSI International Journal of Computer Science Issues, Vol. 8, Issue 5, No 2, September 2011; pp. 259-268.

[17] R.V. Biradar, S. R. Sawant, R. R. Mudholkar, and V.C .Patil, Multihop Routing In Self-Organizing Wireless Sensor Networks, IJCSI International Journal of Computer Science Issues, Vol. 8, Issue 1, January 2011; pp. 155-164.

[18] Y. Liu, Z. Wang, Maximizing energy utilization routing scheme in wireless sensor networks based on minimum hops algorithm, Computers and Electrical Engineering 38 (2012) 703-721.

[19] A. Chamam, S. Pierre, A distributed energy-efficient clustering protocol for wireless sensor networks, Computers and Electrical Engineering 36 (2010) 303-312.

[20] I. Woungang, Wireless ad hoc, sensor and mesh networks, Computers and Electrical Engineering 36 (2010) 259-260.

[21] Nadjib Aitsaadi, Nadjib Achir, Khaled Boussetta, Guy Pujolle, Artificial potential field approach in WSN deployment: Cost, QoM, connectivity, and lifetime constraints, In journal of Computer Networks, 55(1), (2011), pp. 84-105. 
[22] Wen-Hwa Liao, and Hsiao-Hsien Wang, An asynchronous MAC protocol for wireless sensor networks, In Journal of Network and Computer Applications, 31(2008), pp. 807-820

[23] M. Jorgic, I. Stojmenovic, M. Hauspie, and D. Simplot-ryl, Localized algorithms for detection of critical nodes and links for connectivity in ad hoc networks, Proceedings of the 3rd Annual IFIP Mediterranean Ad Hoc Networking Workshop, Turkey, 2004, pp. 360-371.

[24] F. Dai, and J. Wu, An Extended Localized Algorithm for Connected Dominating Set Formation in Ad Hoc Wireless Networks, IEEE Transaction on Parallel and Distributed Systems, 15(10), (2004), pp. 908-920.

[25] G. Wang, G. Cao, T. La Porta, and W. Zhang, Sensor Relocation in Mobile Sensor Networks, in the Proceedings of the 24th Annual Joint Conference of IEEE Computer Communications Societies (INFOCOM'05), Miami, FL, March 2005. 